\title{
Placas anulares diseminadas
}

\section{Disseminated annular plaques}

\author{
Sonia Torres-González, ${ }^{\star}$ Alberto Ramos-Garibay, ${ }^{\ddagger}$ \\ Alfonsina María Decamps-Solano,, Laura Sinaí Parra-Jaramillođ
}

\section{CASO CLÍNICO}

Hombre de 78 años de edad, originario y residente de la Ciudad de México. Sin antecedentes de importancia para su padecimiento actual. Acude a consulta por presentar «ronchas» en las cuatro extremidades desde hace dos años. A la exploración física se observó dermatosis diseminada a extremidades superiores e inferiores, de las primeras afectaba específicamente brazos, antebrazos y dorso de manos, de las segundas muslos y piernas, en forma bilateral y simétrica. Dermatosis de aspecto monomorfo, constituida por eritemas y escamas que confluyen formando incontables placas, de 3 a $4 \mathrm{~mm}$ de diámetro, de bordes bien delimitados,
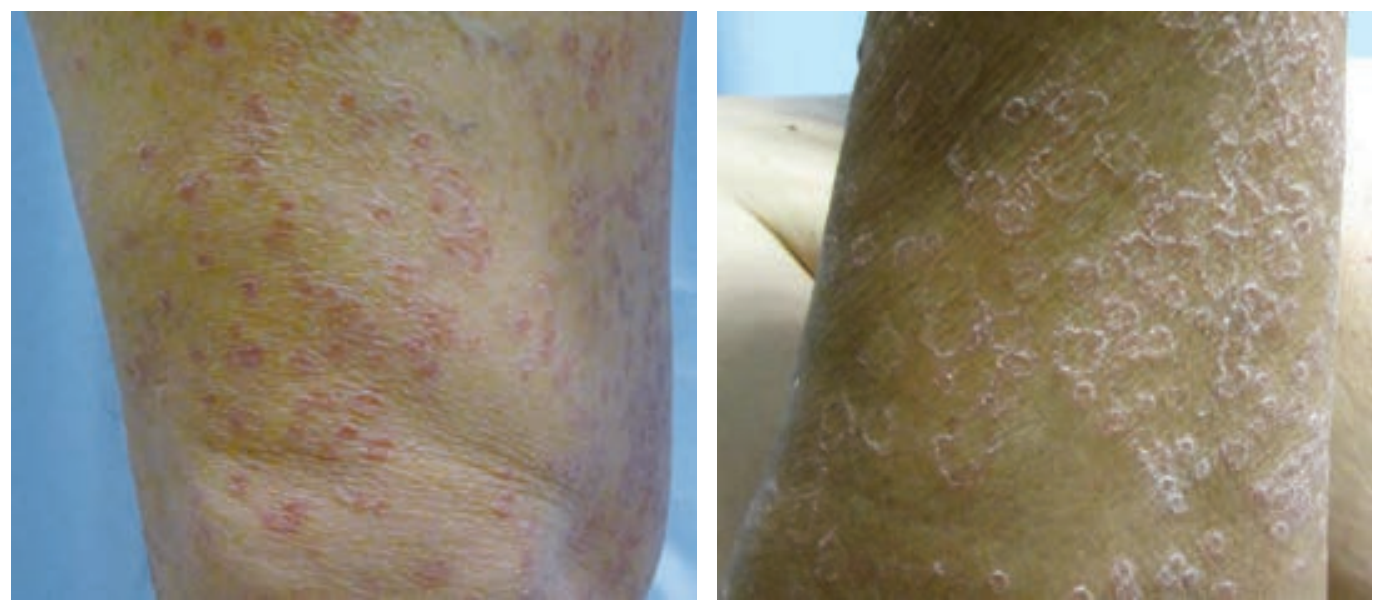

Figura 1:

Aspecto de la dermatosis.

* Médico adscrito al Servicio de Dermatooncología.
₹ Dermatopatólogo.
§ Dermatóloga egresada.
" Médico Residente del segundo año de Dermatología.

Centro Dermatológico «Dr. Ladislao de la Pascua», SSCDMX. México.
Citar como: Torres-González S, Ramos-Garibay A, Decamps-Solano AM, ParraJaramillo LS. Placas anulares diseminadas. Rev Cent Dermatol Pascua. 2021; 30 (3): 170-176. https://dx.doi.org/10.35366/103833 


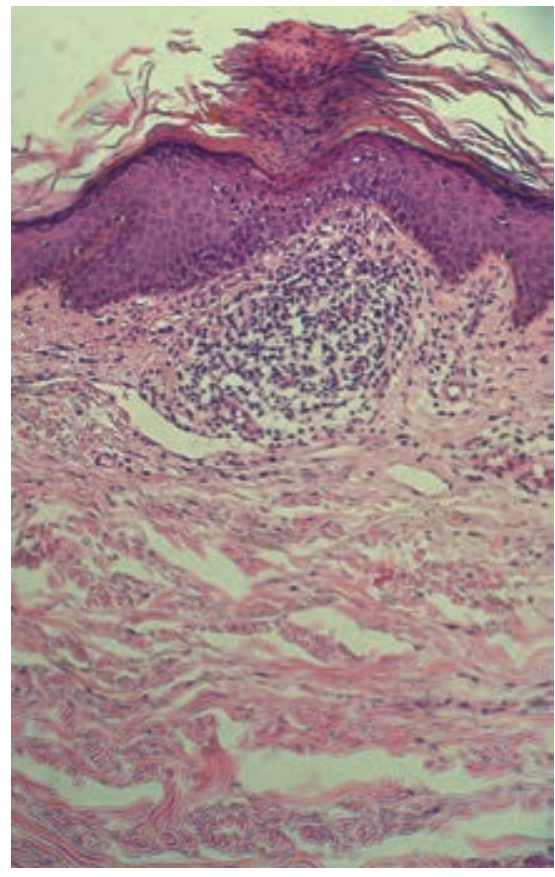

Figura 2:

Presencia de laminilla cornoide

$y$ un infiltrado linfocitario en

dermis superficial (H\&E 10x).

Figura 3:

Por debajo de la laminilla cornoide se observa atrofia de la capa espinosa con queratinocitos de citoplasma claro (H\&E 20x).

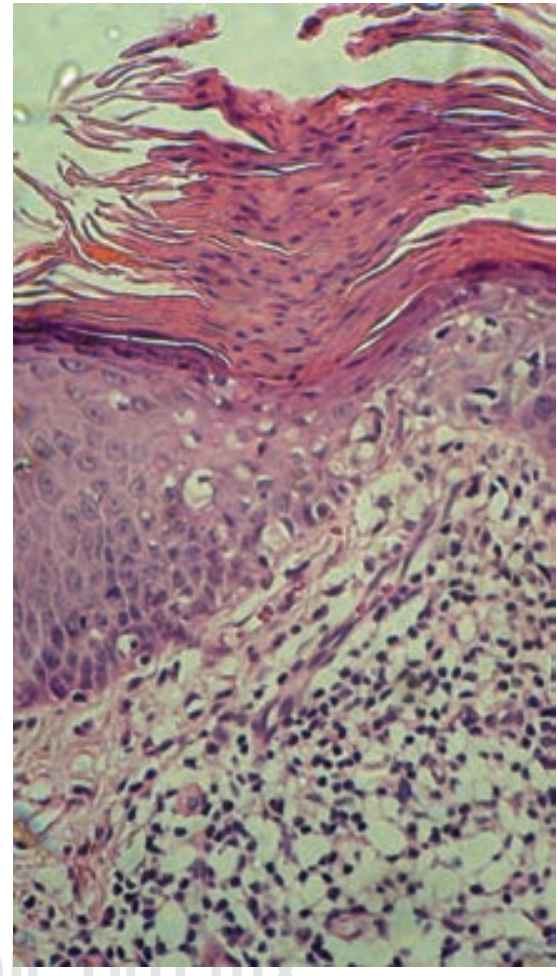

policíclicos, de superficie anfractuosa, con piel de características normales en el centro. De evolución crónica y asintomática (Figura 1).

La biopsia incisional reportó epidermis con áreas de hiperqueratosis paraqueratósica, simulando una laminilla cornoide, áreas de hipergranulosis y atrofia con degeneración hidrópica en esos puntos. La dermis superficial muestra infiltrados linfocitarios perivasculares discretos (Figuras 2 y 3).

Con los datos antes proporcionados, ¿cuál es su diagnóstico? 


\section{DIAGNÓSTICO: POROQUERATOSIS ACTÍNICA DISEMINADA}

\author{
INTRODUCCIÓN
}

Las poroqueratosis $(\mathrm{PQ})$ constituyen un grupo de genodermatosis poco frecuentes que se caracterizan por un trastorno de la queratinización de origen desconocido. ${ }^{1}$

En 1893, Mibelli describió la forma clásica de PQ, ${ }^{2} \mathrm{y}$ desde entonces se han descrito otras variantes, cada una con características clínicas propias que difieren en cuanto a evolución, topografía y morfología. Todas ellas comparten una característica histopatológica en común: la presencia de lámina cornoide que se observa cuando se realiza biopsia de uno de los bordes hiperqueratósicos de las lesiones. Éstas inicialmente están constituidas por pápulas hiperqueratósicas que crecen lentamente en forma centrífuga. Después adquieren aspecto anular, con borde policíclico, queratósico con atrofia central. Aunque generalmente son asintomáticas, no es infrecuente que se presenten acompañadas de prurito..$^{3,4}$

Clásicamente se reconocen seis variantes clínicas, las cuales, según su extensión, se clasifican en localizadas: $P Q$ de Mibelli $(P M), P Q$ lineal $(P L)$ y $P Q$ punctata (PP); y diseminadas: $P Q$ actínica superficial diseminada (PASD), PQ superficial diseminada (PSD) y PQ palmoplantar diseminada (PPPD). ${ }^{5,6}$ De manera adicional se han descrito otros tipos, cuya presentación es poco frecuente, como la $P Q$ psicotrópica, $P Q$ facial, $P Q$ gigante, $P Q$ verrugosa hipertrófica, $P Q$ reticulada y $P Q$ papular pruriginosa eruptiva. ${ }^{3}$ De todas, la PASD es la forma más frecuente. ${ }^{6}$

\section{Epidemiología}

Se trata de una enfermedad poco frecuente, pero su incidencia exacta no se conoce. No se ha encontrado asociación con algún grupo étnico en particular; sin embargo, son escasos los reportes de PM y PASD en individuos de piel oscura. ${ }^{6}$ Por lo general, se desarrolla en piel fotoexpuesta, por lo que se ha propuesto la exposición a radiación ultravioleta (UV) como factor de riesgo. ${ }^{7}$ La prevalencia es similar entre hombres y mujeres, aunque con variaciones dependiendo de la forma clínica, la más frecuente es la PM en hombres y la PASD en mujeres. La edad de inicio tiene relación con la forma clínica. ${ }^{6,7}$ La forma eruptiva de PQ se ha asociado a pacientes con inmunosupresión, malignidad o estados inflamatorios crónicos. ${ }^{8}$ Se ha observado progresión de las lesiones a cáncer de piel de tipo no melanoma en $6.9-30 \%$ de los casos, principalmente hacia carcinoma de células escamosas. ${ }^{8}$

\section{Etiología y fisiopatología}

La etiología de la $P Q$ parece tener origen multifactorial; no obstante, aún no se han esclarecido los mecanismos que le dan origen. El desarrollo de la laminilla cornoide asociada a características displásicas localizadas sugiere origen clonal de queratinocitos anormales. ${ }^{9}$ Esta hipótesis se sustenta en el hecho de que se ha detectado ADN aneuploide en los queratinocitos cercanos a la laminilla cornoide, así como incremento en la proporción de células en fase $S$ y G2/M del ciclo celular, y la expresión aumentada de oncoproteínas p53 y Rb. ${ }^{7}$ La presencia de PQ puede ser indicativa de alteración en la maduración de los queratinocitos, 0 por alteraciones en la queratinopoyesis, ya que se ha demostrado la ocurrencia temprana de apoptosis en los queratinocitos, con interrupción en la expresión de loricrina en las áreas afectadas, lo que indica fallas en la diferenciación terminal de los queratinocitos, que se traduce en ausencia de la capa granulosa epidérmica. ${ }^{10}$

Se ha sugerido la existencia de predisposición genética derivada de la observación de casos familiares de $P Q$. Existe una forma de herencia autosómica dominante con penetrancia variable para la PM, PPPD, PSD y PASD. Con respecto a la PASD, hay cuatro loci implicados (DSAP1 en el locus 12q23.2-24.1, ${ }^{11}$ DSAP2 en el 15q25.1-26.1,12 DSAP3 en el 1p31.3-p31.1 ${ }^{13} \mathrm{y}$ DSAP4 en el 16q24.1-24.3). ${ }^{14}$ Otros estudios indican que los locipara PPPD podrían encontrarse en el brazo largo del cromosoma $12^{15}$ y para PSD en el $18 p 11.3 .{ }^{16}$ Para PM se ha reportado inestabilidad cromosómica en el locus 3p12-14. ${ }^{17}$ En fechas recientes se encontraron mutaciones en el gen que codifica para la mevalonato cinasa en 33 y $16 \%$ de pacientes con PASD familiar y esporádica, respectivamente. Los estudios in vitro realizados al respecto sugieren que esta enzima podría proteger a los queratinocitos de la apoptosis inducida por UVA. ${ }^{17}$ Hace poco también se detectó la duplicación del gen EMILIN2 (gen proapoptótico) en una familia con PM. ${ }^{18}$ Existe un caso reportado de gemelos monocigóticos afectados por PL, lo que apoya la participación de factores genéticos en la patogénesis de esta enfermedad. ${ }^{19}$

Además de la predisposición genética, pueden estar involucrados factores externos como radiación ultravioleta, agentes infecciosos, trauma e inmunosupresión. ${ }^{4}$ Respecto a la PASD, existe evidencia que sugiere su asociación con la radiación UV. ${ }^{7}$ Hay casos de empeo- 
ramiento de las lesiones después de tratamiento con PUVA $^{20}$ y radioterapia. ${ }^{21,22}$ Así mismo, en esta variante clínica de $P Q$, las lesiones se localizan con más frecuencia en áreas fotoexpuestas, y empeoran durante el verano. ${ }^{7}$

Se han reportado casos de lesiones en regiones cercanas a sitios de hemodiálisis ${ }^{23}$ y sobre una cicatriz por quemadura, ${ }^{24}$ lo que sugiere intervención de un fenómeno isomórfico de Koebner en el origen de las lesiones; si bien no se ha demostrado el papel de este factor. ${ }^{4,7}$ También hay casos esporádicos asociados al uso de medicamentos como diuréticos ${ }^{25}$ (furosemida), tratamiento con esteroides locales, ${ }^{26}$ inhibidores de TNF- $\alpha,{ }^{27}$ antibióticos ${ }^{28}$ e hidroxiurea, ${ }^{29}$ observándose regresión de las lesiones tras la suspensión del fármaco. ${ }^{7}$

Está en discusión el papel de la infección por virus del papiloma humano (VPH), ya que se ha aislado a este agente patógeno (tipos 14 y 66) de lesiones de $P Q$ en pacientes postrasplantados. ${ }^{7,30}$

Las deficiencias inmunitarias adquiridas representan $50 \%$ de los casos nuevos. ${ }^{4}$ Hay casos de PQ después de trasplantes renal, hepático, de corazón, de médula ósea y de pulmones, ${ }^{4,31}$ en pacientes con cáncer de origen hematopoyético ${ }^{32}$ y pacientes con infección por VIH concomitante. ${ }^{33}$ También se ha visto asociación con hepatopatías, tumores malignos sólidos (colon, esófago, ovario, próstata, hígado) y enfermedades autoinmunes e inflamatorias (pénfigo, dermatomiositis, artritis reumatoide, diabetes mellitus, psoriasis, asma, síndrome de Sjögren, etc.). ${ }^{7}$

La degeneración de las lesiones hacia neoplasias malignas se ha descrito en todas las formas clínicas de $P Q$, con incidencia entre 7.5 y 11\%;,434,35 pero las variedades $P L$ y $P Q$ gigante son las que tienen la mayor incidencia de transformación maligna. ${ }^{4}$ Hasta el momento, los factores de riesgo identificados para que se desarrolle lo anterior son el tiempo de evolución, el tamaño de las lesiones y la localización en extremidades. Las neoplasias se presentan con más frecuencia en piel no expuesta a luz solar, con un periodo de latencia promedio de 36 años. ${ }^{4}$ El carcinoma de células escamosas es el tipo de malignidad más frecuente. ${ }^{4}$

\section{Características clínicas}

La lesión primaria de la $P Q$ es una pápula de 2-3 mm de diámetro, color marrón, queratósica y generalmente asintomática que se expande de manera centrífuga y después de semanas o meses forma una placa circunscrita, con borde policíclico, que puede variar en forma y tamaño. Su borde rodea una depresión que contiene una laminilla córnea que se dirige hacia el centro de la placa, generalmente más palpable que visible. El centro de la placa es ligeramente atrófico y deprimido, con frecuencia hiperpigmentado. ${ }^{7}$ Como ya se mencionó, de acuerdo con la distribución, tamaño y número de las lesiones, se distinguen varias formas clínicas que se describirán a continuación.

Poroqueratosis de Mibelli. Se caracteriza por la presencia de una o varias placas anulares de gran tamaño, que se presentan más frecuentemente de forma unilateral, con atrofia central, en ocasiones con hiperqueratosis. Predomina en las extremidades, pero hay casos reportados de lesiones en cara, labios, palmas, plantas, genitales, piel cabelluda y mucosa oral. Suele ser asintomática, aunque a veces está acompañada de prurito. Puede ser esporádica o familiar, en cuyo caso inicia en la infancia, más en hombres que en mujeres. ${ }^{7}$

Poroqueratosis actínica superficial diseminada. Es la forma más frecuente. Se presenta más en áreas geográficas con alta exposición solar (Australia, por ejemplo). En general aparece entre la tercera y cuarta décadas de la vida, con patrón de herencia autosómico dominante. Se manifiesta en forma de numerosas lesiones anulares (a veces cientos de ellas), pequeñas (aproximadamente 1 centímetro de diámetro), que se distribuyen de manera bilateral y simétrica en áreas fotoexpuestas, principalmente piernas, antebrazos, hombros y espalda y, con menos frecuencia, en cara. Las lesiones causan prurito en un tercio de los pacientes y confluyen en placas policíclicas que tienden a empeorar después de la exposición al sol o a fuentes artificiales de UVA y UVB. ${ }^{7}$

Poroqueratosis superficial diseminada. Es clínicamente similar a la PASD, pero la edad de inicio es a los 5-10 años y no parece que la radiación ultravioleta juegue un papel importante en la aparición de las lesiones, que suelen ser localizadas tanto en áreas fotoexpuestas como en no fotoexpuestas (tronco, genitales, palmas, plantas). Cursan con prurito en un tercio de los casos. Se ha observado un patrón de herencia autosómico dominante. ${ }^{7}$

Poroqueratosis lineal. Presentación rara, que se manifiesta en forma de placas lineales hiperqueratósicas y unilaterales en extremidades, siguiendo las líneas de Blaschko. Se han descrito formas localizadas, generalizadas y zosteriformes. Se suele asociar con lesiones ungueales. Se presenta a edades tempranas y puede incluso estar presente desde el nacimiento. Es discretamente más frecuente en mujeres. ${ }^{7}$

Poroqueratosis punctata. Se manifiesta con múltiples lesiones puntiformes de 1-2 mm de diámetro, 
en ocasiones dolorosas a la presión, que aparecen al inicio en palmas y plantas y pueden confundirse con las lesiones presentes en la enfermedad de Darier, liquen nítido y queratodermia hereditaria punteada. Puede asociarse con la PM o PL. ${ }^{7}$

Poroqueratosis palmoplantar diseminada. Constituida por pápulas queratósicas de 1-2 mm de diámetro de color rojo-marrón que se distribuyen de manera bilateral y simétrica, inicialmente se ubican en palmas y plantas para después diseminarse a zonas como extremidades y tronco. La mucosa oral puede verse afectada. Predomina en hombres y puede presentarse en la edad adulta o durante la adolescencia. La mayoría de los casos son familiares. ${ }^{7}$

Otras variantes. Hay formas más raras de $P Q$, entre ellas la poroqueratosis reticulada ${ }^{36}$ psicotrópica, ${ }^{37-39}$ eruptiva prurítica ${ }^{40}$ ulcerativa,${ }^{41}$ genital aislada,${ }^{42}$ folicular, ${ }^{43}$ bulosa, ${ }^{44}$ pustular ${ }^{45}$ y la $P Q$ similar a dermatitis seborreica.$^{46} \mathrm{El}$ poroqueratoma se manifiesta como placa o nódulo único, de aspecto más o menos verrugoso, que histológicamente presenta la característica laminilla cornoide. ${ }^{47}$

\section{Histopatología}

La característica distintiva de la PQ es la presencia de la laminilla cornoide, que se puede observar en la periferia de las lesiones, a nivel del borde queratósico, y que se conforma por una columna vertical y estrecha de corneocitos paraqueratósicos, en el contexto de una capa córnea gruesa y ortoqueratósica. Se observa ausencia o disminución de la capa granulosa de la epidermis y la capa espinosa contiene queratinocitos disqueratósicos o vacuolados. Los queratinocitos basales también pueden mostrar vacuolización. La dermis superior muestra infiltrado inflamatorio compuesto principalmente por linfocitos TCD4+. La dermis papilar ocasionalmente contiene eosinófilos, cuerpos coloides o depósitos de material amiloide. En la porción central de las placas se puede observar hiperqueratosis ortoqueratósica leve, con una capa espinosa atrófica. En la dermis se encuentran capilares dilatados rodeados por infiltrado linfocitario moderado. ${ }^{7}$

\section{DIAGNÓSTICO}

El diagnóstico se basa en las características clínicas de las lesiones y el aspecto histopatológico típico. Sin embargo, es necesario tomar en cuenta que la presencia de la laminilla cornoide no es patognomónica de esta entidad, y que puede presentarse también en otros padecimientos, como en la queratosis actínica, carcinoma basocelular y espinocelular, verrugas y queratosis seborreicas, por lo que se deben tomar en cuenta como diagnósticos diferenciales. ${ }^{7}$

\section{TRATAMIENTO}

Hay numerosas terapias tópicas, sistémicas y quirúrgicas para la $\mathrm{PQ}$. Los análogos de la vitamina $\mathrm{D} 3$ tópicos son utilizados en numerosos reportes de caso, con mejoría de las lesiones en la PASD, con pocos o ningún efecto adverso; no obstante, con mala respuesta en el caso de la PL. ${ }^{3}$ El mecanismo de acción exacto aún no se conoce.

Se observan buenos resultados con el uso de retinoides en pacientes con PL (acitretina oral, tretinoína tópica), en formas diseminadas como en la poroqueratosis diseminada gigante (acitretina oral), en la PPPD (acitretina oral), poroqueratosis genital (acitretina oral), poroqueratosis psicotrópica (isotretinoína oral) y PM (tretinoína tópica, etretinato oral), con efectos más modestos en poroqueratosis facial y PASD. ${ }^{3}$ El imiquimod tópico dio muy buenos resultados en varios reportes de casos de PM, y en algunas comunicaciones aisladas de PASD y PL. ${ }^{3}$ El tratamiento con 5-fluorouracilo muestra resultados heterogéneos que van desde «modestos" hasta «excelentes» en diferentes formas clínicas de $P Q$, y se utilizaron en combinación para tratar con éxito un caso de $\mathrm{PM}^{3}{ }^{3}$

Existen pocos reportes en la literatura acerca del uso de agentes inmunosupresores; pero, pueden resultar benéficos para disminuir las molestias asociadas, como el prurito y la inflamación, aunque con poca mejoría de las lesiones. En este apartado se puede mencionar el uso de esteroides sistémicos y tópicos e inhibidores de calcineurina, solos o en combinación. ${ }^{3}$ También se ha empleado tratamiento con láser y fototerapia, con resultados variables. ${ }^{3}$ Dentro de las intervenciones quirúrgicas que se utilizan se encuentran la dermoabrasión, escisión completa de las lesiones, cirugía por radiofrecuencia, entre otras. Por último, la crioterapia se emplea con éxito para tratar lesiones anogenitales y en labios, así como en la PL, con modesta mejoría en la PASD. ${ }^{3}$

Si bien la cantidad de opciones terapéuticas que se emplean es bastante amplia, hasta el momento no se han realizado ensayos clínicos aleatorizados, por lo que no existen guías nacionales o internacionales para normar la conducta terapéutica. En 2017, Weidner y colaboradores efectuaron una revisión sistemática acerca del tratamiento de la $P Q$, incluyendo en total 
88 artículos, principalmente reportes y series de casos, no se encontraron ensayos clínicos aleatorizados. La revisión concluye que para la PM hay buenos resultados con el tratamiento con imiquimod al $5 \%$ y puede considerarse como alternativa la crioterapia en caso de lesiones pequeñas. La PL respondió bien con retinoides tópicos y sistémicos. El tratamiento con análogos de la vitamina D3 podría ser la mejor opción para las formas diseminadas, y la crioterapia y las intervenciones quirúrgicas las opciones más viables para las áreas donde el uso de agentes tópicos es difícil o están contraindicados. ${ }^{3}$

\section{COMENTARIO}

El objetivo de este trabajo es describir el caso de un paciente que se presentó con las lesiones típicas de $P Q$ actínica superficial diseminada, entidad rara en la población general. La enfermedad se desarrolló en un hombre de 78 años, lo que la hace aún más inusual por el grupo de edad y el sexo al que pertenece.

Debido a las implicaciones del diagnóstico, sobre todo tomando en cuenta el pronóstico y su asociación con neoplasias malignas, es de vital importancia realizar un diagnóstico oportuno y brindar tratamiento y seguimiento adecuados a estos pacientes. Como ya se comentó, existe una amplia variedad de modalidades terapéuticas reportadas en la literatura, aunque sin un consenso basado en ensayos clínicos aleatorizados que permitan estandarizar el tratamiento. Dado que es una entidad poco frecuente y con muchas variedades clínicas, resulta difícil reunir a un número aceptable de pacientes para efectuar diferentes estudios y esclarecer la fisiopatología de la enfermedad, y aún más, para poder llevar a cabo los ensayos clínicos necesarios para establecer la efectividad y seguridad de las diversas opciones terapéuticas.

El paciente, cuyo caso fue el motivo de publicación de este artículo, fue tratado con 5-fluorouracilo en crema, aplicación diaria por la noche, por ocho semanas, con lo cual se obtuvo una remisión adecuada, en la actualidad sin lesiones activas; aun así, continúa en observación en el área de dermatología general.

\section{REFERENCIAS}

1. Medina CD, Ramos GJA, Cantú CPP. Poroqueratosis actínica superficial. Presentación de un caso. Rev Cent Dermatol Pascua. 2005; 14: 81-86.

2. Mibelli V. Contributo allo studio della ipercheratosi dei canali sudoriferi (porocheratosi). G Ital Mal Ven Pelle. 1893; 28: 313-355.
3. Weidner T, Illing T, Miguel D, Elsner P. Treatment of porokeratosis: a systematic review. Am J Clin Dermatol. 2017; 18: 435-449.

4. Sertznig $P$, von Felbert V, Megahed M. Porokeratosis: present concepts. J Eur Acad Dermatol Venereol. 2012; 26: 404-412. doi: 10.1111/j.1468-3083.2011.04275.x.

5. Gu CY, Zhang CF, Chen LJ, Xiang LH, Zheng ZZ. Clinical analysis and etiology of porokeratosis. Exp Ther Med. 2014; 8: 737-741.

6. Isac L, Jiquan S, Kumar H, Pottapulan S. Porokeratoses-A comprehensive review. EJPMR. 2018; 5: 544-554.

7. Kanitakis J. Porokeratoses: an update of clinical, aetiopathogenic and therapeutic features. Eur J Dermatol. 2014; 24: 533-544.

8. Williams GM, Fillman EP. Porokeratosis. In: StatPearls [Internet]. Treasure Island (FL): StatPearls Publishing; 2021. Available in: https:// www.ncbi.nlm.nih.gov/books/NBK532290/

9. Reed RJ, Leone P. Porokeratosis--a mutant clonal keratosis of the epidermis. I. Histogenesis. Arch Dermatol. 1970; 101: 340-347.

10. Shen CS, Tabata K, Matsuki M, Goto T, Yokochi T, Yamanishi K. Premature apoptosis of keratinocytes and the dysregulation of keratinization in porokeratosis. Br J Dermatol. 2002; 147: 498-502.

11. Xia JH, Yang YF, Deng H, Tang BS, Tang DS, He YG et al. Identification of a locus for disseminated superficial actinic porokeratosis at chromosome 12q23.2-24.1. J Invest Dermatol. 2000; 114: 1071-1074.

12. Xia K, Deng H, Xia JH, Zheng D, Zhang HL, Lu CY et al. A novel locus (DSAP2) for disseminated superficial actinic porokeratosis maps to chromosome 15q25.1-26.1. Br J Dermatol. 2002; 147: 650-654.

13. Liu $P$, Zhang S, Yao Q, Liu X, Wang X, Huang $C$ et al. Identification of a genetic locus for autosomal dominant disseminated superficial actinic porokeratosis on chromosome 1p31.3-p31.1. Hum Genet. 2008; 123: 507-513.

14. Luan J, Niu Z, Zhang J, Crosby ME, Zhang Z, Chu X et al. A novel locus for disseminated superficial actinic porokeratosis maps to chromosome 16q24.1-24.3. Hum Genet. 2011; 129: 329-334.

15. Wei SC, Yang S, Li M, Song YX, Zhang XQ, Bu L et al. Identification of a locus for porokeratosis palmaris et plantaris disseminata to a 6.9-cM region at chromosome 12q24.1-24.2. Br J Dermatol. 2003; 149: 261-267.

16. Wei S, Yang S, Lin D, Li M, Zhang X, Bu L et al. A novel locus for disseminated superficial porokeratosis maps to chromosome 18p11.3. J Invest Dermatol. 2004; 123: 872-875.

17. Zhang SQ, Jiang T, Li M, Zhang X, Ren YQ, Wei SC et al. Exome sequencing identifies MVK mutations in disseminated superficial actinic porokeratosis. Nat Genet. 2012; 44: 1156-1160.

18. Scappaticci S, Lambiase S, Orecchia G, Fraccaro M. Clonal chromosome abnormalities with preferential involvement of chromosome 3 in patients with porokeratosis of Mibelli. Cancer Genet Cytogenet. 1989; 43: 89-94.

19. Guillot P, Taieb A, Fontan I, Bilhou-Nabera C, Viard E, Renaud P et al. Linear porokeratosis of Mibelli in monozygotic twin girls. Ann Dermatol Venereol. 1991; 118: 519-524.

20. Allen AL, Glaser DA. Disseminated superficial actinic porokeratosis associated with topical PUVA. J Am Acad Dermatol. 2000; 43: 720-722.

21. Romaní J, Pujol RM, Casanova JM, de Moragas JM. Disseminated superficial porokeratosis developing after electron-beam total skin irradiation for mycosis fungoides. Clin Exp Dermatol. 1996; 21: 310-312. 
Torres-González S y cols. Placas anulares diseminadas

22. Batchelor JM, Fife K, Burrows NP: Localized porokeratosis secondary to ionizing radiotherapy for prostate carcinoma. Arch Dermatol. 2010; 146: 1318-1320.

23. Nakazawa A, Matsuo I, Ohkido M. Porokeratosis localized to the access region for hemodialysis. J Am Acad Dermatol. 1991; 25: 338-340.

24. Nova MP, Goldberg LJ, Mattison T, Halperin A. Porokeratosis arising in a burn scar. J Am Acad Dermatol. 1991; 25: 354-356.

25. Kroiss MM, Stolz W, Hohenleutner U, Landthaler M. Disseminated superficial porokeratosis induced by furosemide. Acta Derm Venereol. 2000; 80: 52-53.

26. Yazkan F, Turk BG, Dereli T, Kazandi AC. Porokeratosis of Mibelli induced by topical corticosteroid. J Cutan Pathol. 2006; 33: 516-518.

27. Stewart $L$, Howat $A$, Coulson I. Disseminated superficial porokeratosis secondary to immunosuppression induced by etanercept for extensive psoriasis. Arch Dermatol. 2010; 146: 1193-1194.

28. Goulding JM, Teoh JK, Carr RA, Humphreys F, Gee BC. Eruptive disseminated superficial porokeratosis with rapid resolution: a druginduced phenomenon? Clin Exp Dermatol. 2009; 34: 895-897.

29. Kanitakis J, Arbona-Vidal E, Faure M. Porokeratosis in patients with polycythemia rubra vera: a new side effect of hydroxyurea? J Eur Acad Dermatol Venereol. 2012; 26: 1040-1041.

30. Esser AC, Pittelkow MR, Randle HW. Human papillomavirus isolated from transplant-associated porokeratoses of Mibelli responsive to topical 5\% imiquimod cream. Dermatol Surg. 2006; 32: 858-861.

31. Macmillan AL, Roberts SO. Porokeratosis of Mibelli after renal transplantation. Br J Dermatol. 1974; 90: 45-51.

32. Luelmo-Aguilar J, Gonzalez-Castro U, Mieras-Barcelo C, CastellsRodellas A. Disseminated porokeratosis and myelodysplastic syndrome. Dermatology. 1992; 184: 289.

33. Rodríguez EA, Jakubowicz S, Chinchilla DA, Carril A, Viglioglia PA. Porokeratosis of Mibelli and HIV-infection. IntJ Dermatol. 1996; 35: $402-404$.

34. Goerttler EA, Jung EG. Porokeratosis [correction of Parakeratosis] Mibelli and skin carcinoma: a critical review. Humangenetik. 1975; 26: 291-296.

35. Otsuka F, Someya T, Ishibashi Y. Porokeratosis and malignant skin tumors. J Cancer Res Clin Oncol. 1991; 117: 55-60.
36. Helfman RJ, Poulos EG. Reticulated porokeratosis. A unique variant of porokeratosis. Arch Dermatol. 1985; 121: 1542-1543.

37. McGuigan K, Shurman D, Campanelli C, Lee JB. Porokeratosis ptychotropica: a clinically distinct variant of porokeratosis. J Am Acad Dermatol. 2009; 60: 501-503.

38. Yeo J, Winhoven S, Tallon B. Porokeratosis ptychotropica: a rare and evolving variant of porokeratosis. J Cutan Pathol. 2013; 40: 1042-1047.

39. Corradin MT, Giulioni E, Forcione M, Fiorentino R, Faggion D, Alaibac Metal. Porokeratosis ptychotropica. Eur J Dermatol. 2011;21: 416-417.

40. Kanzaki T, Miwa N, Kobayashi T, Ogawa S. Eruptive pruritic papular porokeratosis. J Dermatol. 1992; 19: 109-112.

41. Watanabe T, Murakami T, Okochi H, Kikuchi K, Furue M. Ulcerative porokeratosis. Dermatology. 1998; 196: 256-259.

42. Chen TJ, Chou YC, Chen CH, Kuo TT, Hong HS. Genital porokeratosis: a series of 10 patients and review of the literature. $\mathrm{Br} J$ Dermatol. 2006; 155: 325-329.

43. Wang NS, Gruson LM, Kamino H. Facial follicular porokeratosis: a case report. Am J Dermatopathol. 2010; 32: 720-722.

44. Ricci C, Rosset A, Panizzon RG. Bullous and pruritic variant of disseminated superficial actinic porokeratosis: successful treatment with grenz rays. Dermatology. 1999; 199: 328-331.

45. Miller DD, Ruben BS. Pustular porokeratosis. J Cutan Pathol. 2009; 36: 1191-1193.

46. Asawanonda P, Noppakun N, Huiprasert P. Seborrheic keratosis-like porokeratosis: a case report. Dermatol Online J. 2005; 11: 18.

47. Walsh SN, Hurt MA, Santa Cruz DJ. Porokeratoma. Am J Surg Pathol. 2007; 31: 1897-1901.

\section{Correspondencia:}

Dra. Sonia Torres-González

Dr. Vértiz No. 464, esq. Eje 3 Sur,

Col. Buenos Aires, 06780,

Alcaldía Cuauhtémoc, CDMX

Tel: 55 5519-6351

E-mail: sonia220593@yahoo.com.mx 